\title{
Pneumoencephalography in the workup of neuropsychiatric illnesses: a historical perspective
}

\author{
Mariam Ishaque, PhD, ${ }^{1}$ David J. Wallace, MD, ${ }^{2}$ and Ramesh Grandhi, MD² \\ ${ }^{1}$ Long School of Medicine and ${ }^{2}$ Department of Neurosurgery, University of Texas Health at San Antonio, Texas
}

Throughout history, neurosurgical procedures have been fundamental in advancing neuroscience; however, this has not always been without deleterious side effects or harmful consequences. While critical to the progression of clinical neuroscience during the early 20th century, yet, at the same time, poorly tolerated by patients, pneumoencephalography is one such procedure that exemplifies this juxtaposition. Presented herein are historical perspectives and reflections on the role of the pneumoencephalography in the diagnosis and treatment of neuropsychiatric illnesses.

https://thejns.org/doi/abs/10.3171/2017.6.FOCUS17238

KEY WORDS pneumoencephalography; ventriculography; history; neuropsychiatric illness

$\mathrm{T}$ HROUGHOUT history, neurosurgical interventions have been crucial, and at times controversial, in their attempts to better understand, diagnose, and treat neuropsychiatric conditions. Neuroradiological techniques have likewise been fundamental in the advancement of clinical neuroscience and our ability to manage intracranial pathology. Pneumoencephalography (PEG) is a technique that bridges the realms of both neurosurgery and neuroradiology. Performed extensively for a period of about 50 years, PEG has a peculiar history in neuropsychiatry and, now, an ambiguous legacy. Herein, we focus on the development, applications, cultural relevance, and role of PEG in the workup of neuropsychiatric illnesses.

\section{History of Pneumoencephalography}

Soon after Wilhelm Röntgen's discovery of x-rays in 1895 , the skull radiograph emerged as the first neuroradiological method. This was the primary means to diagnose CNS structural pathology in the early 20th century. However, due to the limited soft-tissue contrast provided by the skull radiograph, clinicians were hopeful to use contrast media in conjunction with radiography to enhance anatomical depiction. Walter Dandy, a neurosurgical resident at the Johns Hopkins Hospital, hypothesized that compounds used for retrograde pyelography (e.g., potassium iodide, bismuth subnitrate, argyrol) could be suitable for intracranial contrast. ${ }^{33} \mathrm{He}$ tested his hypotheses in canines through intraventricular injection of these compounds. The radiopaque media turned out to be toxic to the animals, and ultimately, fatal. Soon after, Dandy incidentally observed a patient with a penetrating head injury to also have pneumocephalus. This fortuitous event prompted Dandy to develop what would later be known as ventriculography in 1918, a procedure that entailed replacing CSF with air to provide contrast in the ventricular system (Fig. 1). ${ }^{7}$ Air was typically introduced through puncture of an open fontanel or via a bur hole. ${ }^{9,18,33}$ Soon after the development of ventriculography, Dandy realized that air from ventriculograms often escaped onto the surface of the brain. Using this observation, he developed the pneumoencephalogram in $1919 .{ }^{6}$ In this novel technique, CSF was replaced by air in the spinal subarachnoid space, allowing for better resolution of intracranial structures and, consequently, improved diagnosis of intracranial lesions and CSF flow abnormalities (Fig. 2). ${ }^{12,20}$ Dandy had concerns about the risk of herniation in patients with increased intracranial pressure and thus tended to prefer ventriculography for his patients. ${ }^{12}$ The use of PEG nevertheless increased as Dandy's work was replicated and implemented in other hospitals, particularly by a contemporary German internist at the City Hospital of Brunswick, Adolf Bingel. ${ }^{33}$ In fact, Bingel is considered by some to be the "second inventor" of lumbar pneumoencephalography. ${ }^{26}$

Several aspects of PEG limited its rapid and widespread 


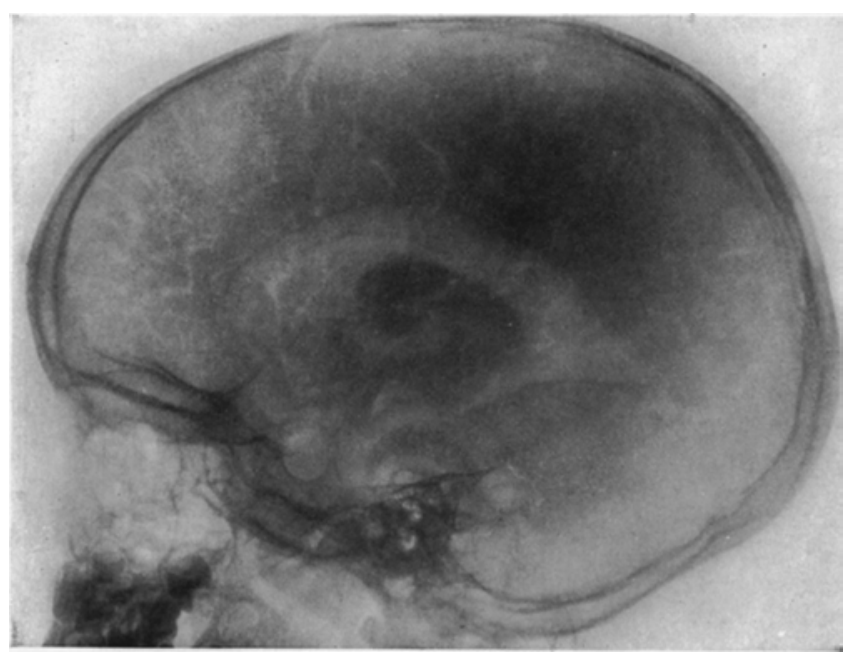

FIG. 1. Ventriculogram of the head after injection of air into a lateral ventricle. The air has passed out of the ventricular system and filled the cerebral sulci, which appear as a network of lines. The cisterna interpeduncularis and the major branches passing to the cerebral sulci are fairly distinct just above the sella turcica. The lateral ventricle is normal. Reproduced from Dandy WE: Röntgenography of the brain after the injection of air into the spinal canal. Ann Surg 70:397-403, 1919.

adoption; it was notorious for its invasiveness, poor patient tolerance, and potentially severe complications. Side effects commonly included severe headaches, nausea, and vomiting. White and colleagues reported on short-term clinical sequelae following PEG among 50 patients. Over the course of 7 days, nearly $80 \%$ complained of headaches, 34\% had neck stiffness, 34\% experienced vomiting, and nearly $75 \%$ were tachycardic. ${ }^{35}$ Furthermore, the procedure became more cumbersome as it required patients to be manipulated into various positions while strapped to a table to image air traversing the ventricles and subarachnoid space. ${ }^{12}$ By some neurosurgeons' accounts, mortality was reported to be as high as $30 \% .^{20} \mathrm{PEG}$ additionally suffered from low resolution and relatively nonspecific findings. Consistency in interpretation and standardized reporting of studies was challenging, particularly due to the lack of a robust control group, as the procedure was too invasive and risky to obtain images in healthy patients. ${ }^{34}$ In fact, in 1929, the American Roentgen Ray Society decreed that it was inappropriate to use healthy patients as control subjects for PEG studies. To mitigate this, data from psychiatric patients often served as control data when studying CNS tumors or hydrocephalus, as these patients were thought to be without any macrostructural pathology.

Despite its low resolution and high potential for morbidity and mortality, PEG played an important role in neurological and psychiatric diagnostics for nearly half a century; that is, until it became virtually obsolete with the advent of angiography in the 1950s and CT imaging in the 1970s. ${ }^{12}$

\section{Schizophrenia}

The most widely reported applications of PEG to psychiatric disorders relate to schizophrenia. Clinicians and researchers investigated for structural pathology common

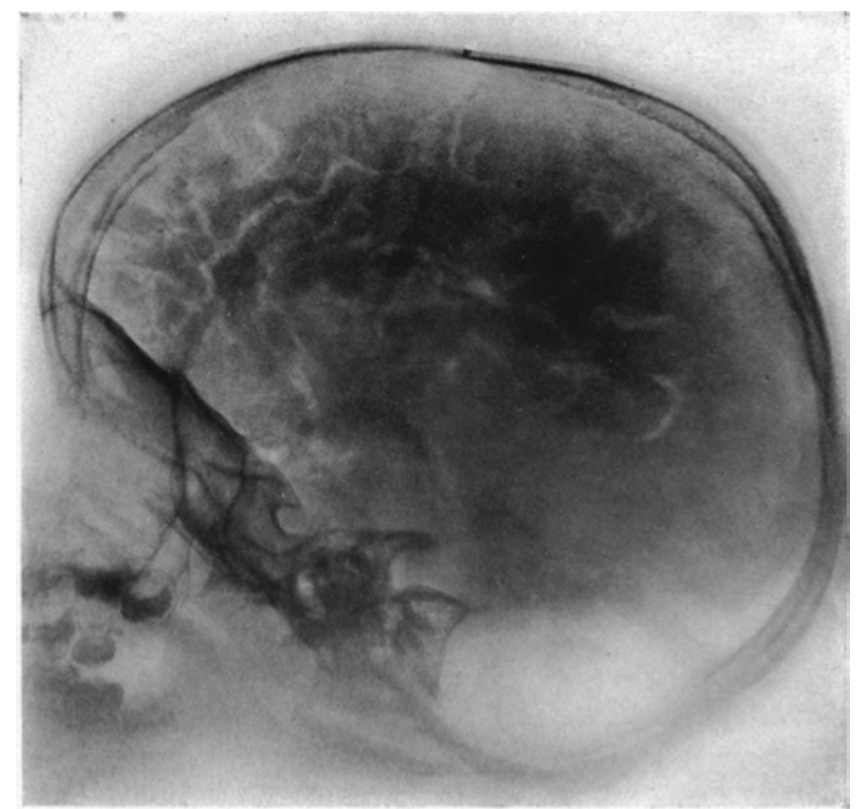

FIG. 2. Pneumoencephalogram of the head after intraspinous injection of air. The sulci and cisterna are more distinct than after the intraventricular injection as shown in Fig. 1. Reproduced from Dandy WE: Röntgenography of the brain after the injection of air into the spinal canal. Ann Surg 70:397-403, 1919.

to schizophrenic patients in hopes of improving time to diagnosis and finding potential therapeutic targets. Several large studies hailed from psychiatrists in western European countries, particularly Germany. Bingel's role in the development and clinical use of PEG likely contributed to the relatively large number of German psychiatrists, neurologists, and neuroradiologists employing PEG - with the help of neurosurgeons-in both research and clinic practice. ${ }^{34}$ The initial studies of the German psychiatrists Walter Jacobi and H. Winkler in schizophrenics demonstrated a high prevalence of cortical and subcortical abnormalities. ${ }^{15}$ The most consistent findings included ventricular enlargement, cortical atrophy in chronic subgroups, and a poorer prognosis associated with cortical atrophy. ${ }^{11,34}$ These studies were among the earliest to suggest that the pathophysiology of schizophrenia involved structural brain changes, such as ventricular dilation or dysmorphia. ${ }^{34}$ They further prompted several other investigations into schizophrenia, which largely corroborated Jacobi and Winkler's conclusions while also providing novel information.

In a series of studies throughout the 1950s at the University of Bonn, Huber reported ventriculomegaly, most often affecting the third ventricle, in 133 of 195 schizophrenic patients. ${ }^{13}$ In 1975, Huber and colleagues published a longitudinal study in which pneumoencephalograms were reacquired in 27 patients from their original cohort. ${ }^{14}$ They found that clinical progression of disease corresponded to more abnormal PEG features. ${ }^{14}$ Importantly, this introduced the concept of using objective, imaging-based measures for prognostic determinations and disease-monitoring purposes, along with advancing knowledge of the natural history of schizophrenia. The first studies in schizophrenia using CT imaging substantiated pneumoencephalographic 
findings of ventriculomegaly. ${ }^{17,27}$ Subsequent investigations demonstrated that ventricular enlargement might be detectable quite early in the disease process, facilitating earlier suspicion and therapeutic intervention in schizophrenic patients. ${ }^{29}$

Ventricular enlargement initially seen via PEG has become pathognomonic in the neuropathological analysis of schizophrenia, confirmed over decades with autopsy as well as more recently with CT and MRI. ${ }^{1}$ The fine detail offered by modern MRI techniques, including volumetric analysis and tractography, has advanced understanding of the neuropathology. Ventricle size has been shown on meta-analysis to average about $26 \%$ greater than agematched controls. ${ }^{28}$ More subtle differences, including reduction in size of the hippocampus, parahippocampal gyrus, temporal lobe, and superior temporal gyrus, have also been shown on MR studies. ${ }^{4,19}$ Further still, diffusion tensor imaging has led to discoveries of increased diffusivity in the superior temporal gyrus, parahippocampal gyrus, and insula. ${ }^{25}$ Ultimately, MRI is elucidating and expanding the seminal findings seen on PEG decades prior.

Early imaging studies using PEG in schizophrenia were thus quite informative and remarkably concordant, despite being limited by a lack of standardized technical and interpretive measures, and often, absence of an appropriate control group. In a wider context, they also challenged the prevailing notion of autopsy as the gold standard for evaluation of neuropathology, as PEG demonstrated microstructural pathology absent on postmortem examination. ${ }^{34}$ Considerable diagnostic impact was not reached, however, due to the previously mentioned limitations. The diagnosis of schizophrenia appeared to remain chiefly clinical, with most psychiatrists using established criteria or "staff consensus" to reach a diagnosis. PEG appeared to occasionally have an adjunct role when the clinical diagnosis was uncertain. ${ }^{34}$

\section{Homosexuality}

At a time when homosexuality was considered an illness, Rudolf Lemke, a German psychiatrist and neurologist at the University of Jena, argued that the etiology was endocrinological in nature and specifically localized to the brain. In his book published in 1940, Lemke used PEG to report decreases in brainstem size in homosexual men, which he attributed to regional disturbances in hormonal activity. He believed that these changes were associated with the subject's "perversion," manifesting as aberrant sexual preferences. ${ }^{23}$ It is unclear how Lemke's theory was received by his contemporaries, but in the years that followed, homosexuality was perceived more so as a remediable illness. As such, a rise in "therapeutic" techniques, both behavioral based (such as psychotherapy) and psychosomatic based (such as electrical shock aversion and icepick hypothalotomies), was seen. ${ }^{30}$ Lemke's endocrinological hypothesis nevertheless represents one of the earliest attempts to identify a biological basis for homosexuality.

\section{Pediatric Neurological Conditions}

Applications of PEG in children and adolescents

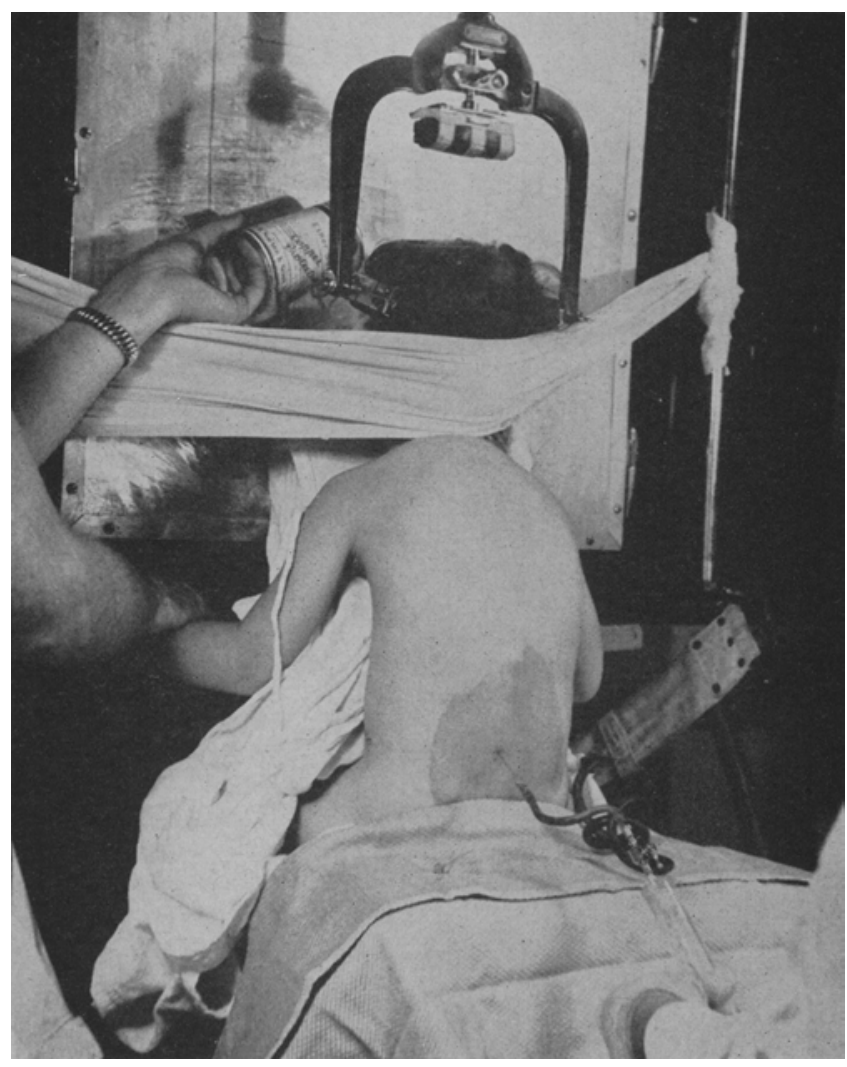

FIG. 3. Photograph from an article outlining technical details of the pediatric PEG. The technique included using atropine and codeine for premedication, with Avertin and ether administered throughout the procedure. The child was positioned sitting on a bench with thighs fastened to the bench. The forehead rested on the vertical Potty-Bucky diaphragm with several pillows and a sandbag on his or her lap built up to rest the chin. This group advocated the use of oxygen instead of room air for injection. Reproduced with permission from Elsevier: Casamajor L, Laidlaw RW, Kozinn PJ: The technique of pneumoencephalography in children: comparative results with air and oxygen injection. J Pediatr 38:463-467, 1951.

emerged comparatively late after introduction of the procedure, likely due to fear of adverse effects on the maturing brain. Once deemed to be tolerable by children, PEG was used to image structural neuropathology in this population (Fig. 3), facilitating examination of both congenital and acquired conditions affecting the CNS (Fig. 4), which most often included workup of hydrocephalus and intracranial tumors. Although several studies used PEG for characterization of pediatric neurological disorders, it was chiefly used for pictorial representation of structural disease and thus, oftentimes, did not directly contribute to the diagnostic process. ${ }^{3}$ Nevertheless, improved visualization of known pathology and demonstration of novel pathology by PEG were indirectly impactful in the long term. They prompted investigations in basic and clinical neuroscience and stimulated discussion among clinicians attempting to explain their findings. ${ }^{3}$ However, some institutions sought to limit the use of PEG in children. By the mid-1950s, the Pediatric Service at the New York Hospital, Cornell Medical Center, had largely restricted the use of PEG 1) to the study of patients with mental retardation in order to dem- 

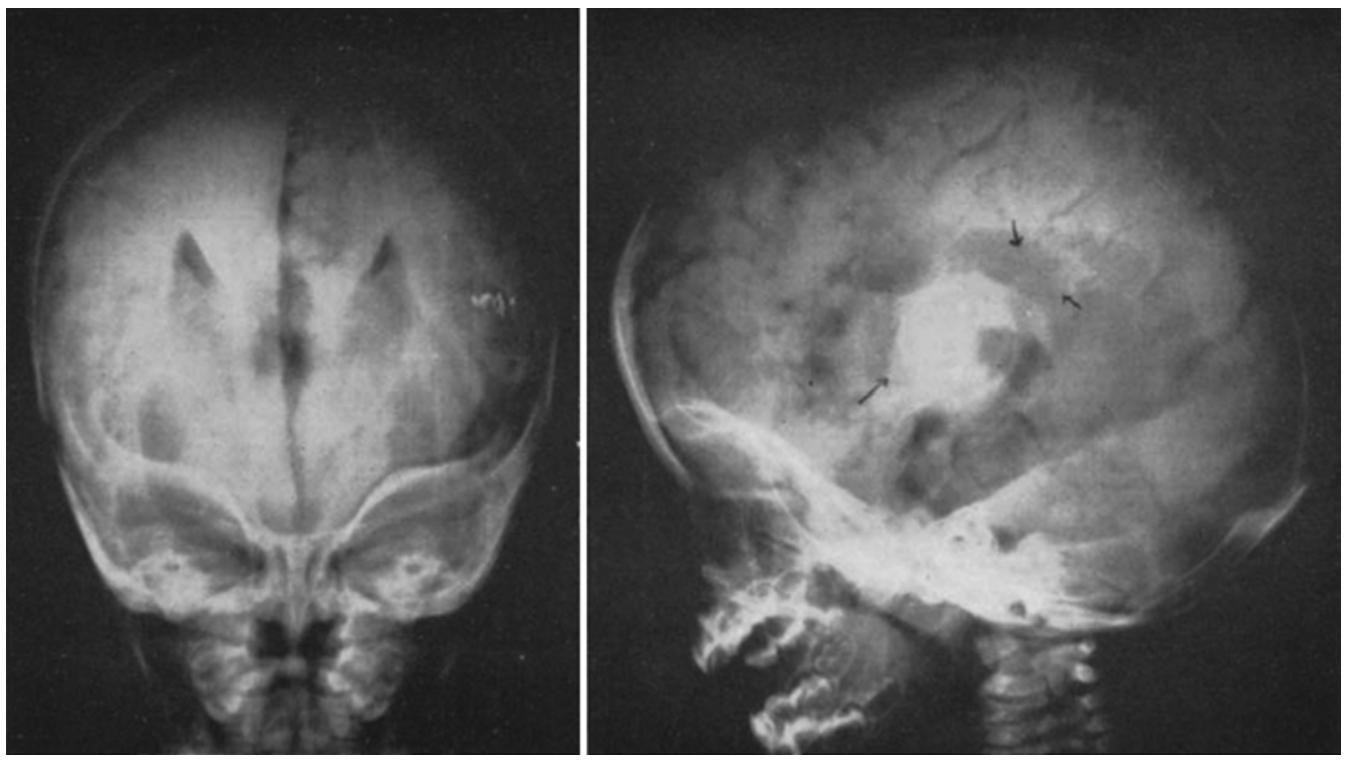

FIG. 4. Pneumoencephalogram demonstrating complete agenesis of the corpus callosum. Separation of the lateral ventricles, pointing of their dorsal margins, concavity of their medial borders, and marked dilation and elevation of the third ventricle in the sagittal view are seen. A dilated interventricular foramen (single arrow) and radially arranged sulci (double arrows) extend through the zone of the corpus callosum. Reproduced with permission from the Radiological Society of North America: Echternacht AP, Campbell JA: Mid-line anomalies of the brain: their diagnosis by pneumoencephalography. Radiology 46:119-131, 1946.

onstrate extent of cerebral atrophy, and 2) to those with idiopathic epilepsy in order to search for an underlying cerebral lesion. This protocol was driven by the perceived extraordinary risks of the procedure. ${ }^{5}$

Eventually, PEG was used in Europe and North America to study children with developmental disorders or abnormal behaviors, with the most prominent application in patients with autism. In 1965, pediatric neurologist Melchior and colleagues at the Rigshospitalet in Copenhagen, Denmark, found "mild" pneumoencephalographic abnormalities in nearly all autistic children in their cohort when compared with typically developing controls. ${ }^{24}$ These abnormalities consisted of an increased Evans ratio (frontal horn width/transverse inner skull diameter), enlargement of the third ventricle, or widened cortical sulci. In 1975, Hauser and colleagues from the Pediatric Neurology Unit of Harvard Medical School and Boston University described pneumoencephalographic findings in 18 children with delayed language development and autistic behaviors. ${ }^{10}$ Although none had been diagnosed with a specific neurological disorder at the time of the study, consistent abnormalities in PEG findings across the cohort suggested a common pathophysiological process: enlargement of the left temporal horn of the lateral ventricle was found in 15 of 18 cases (Fig. 5)..$^{10}$ Of note, prior PEG studies had not directly commented on temporal horn structure. Nevertheless, dilation of the temporal horn indicated selective atrophy of the left cerebral hemisphere in children with autistic behaviors. The most prominent region of localized tissue loss was in the left medial temporal lobe, which led the authors to suspect that medial temporal lobe dysfunction could contribute to the pathogenesis of autism. ${ }^{10}$ They related the behaviors and characteristics of their cohort to those of patient populations with known medial temporal lobe dysfunction, such as Korsakoff syndrome and KlüverBucy syndrome. In this manner, findings derived from PEG supported a neurobiological explanation of autism, offering an alternative to theories of its etiology related to maternal neglect and other psychogenic and environmental factors. ${ }^{10}$

\section{Therapeutic Pneumoencephalography}

For some physicians, PEG represented a therapeutic intervention for neuropsychiatric diseases in addition to serving a diagnostic purpose. The main therapeutic indications included meningitis, epilepsy, and dementia. Adolf Bingel prominently reported on positive effects observed post-PEG. He appreciated defervescence, increased alertness, and an overall improvement in the mental status of patients with bacterial meningitis. ${ }^{26}$ Similar accounts of increased psychomotor activity and alertness in patients with Alzheimer's disease were reported by Schaldach in $1942 .{ }^{31}$ In one account by an unknown physician, injection of air into the subarachnoid space of a comatose patient stimulated near-complete reversal of his impaired consciousness. The therapeutic effect was thought to stem from the introduction of air itself.

Bingel reportedly considered PEG to be "life-saving" in status epilepticus. ${ }^{2}$ Over the next several years, the therapeutic use of PEG in epilepsy was notably recognized by other European neurologists as well. The Austrian epileptologist Felix Frisch acknowledged its controversial, yet effective, role in epilepsy in his book Die Epilepsie, Biologie, Klinik, Therapie in $1937 .{ }^{16}$ Polish neurologist Schleier emphasized its efficacy in, specifically, childhood epilepsy after reporting decreased frequency of seizures post-PEG in 49 children whom she followed for over 3 


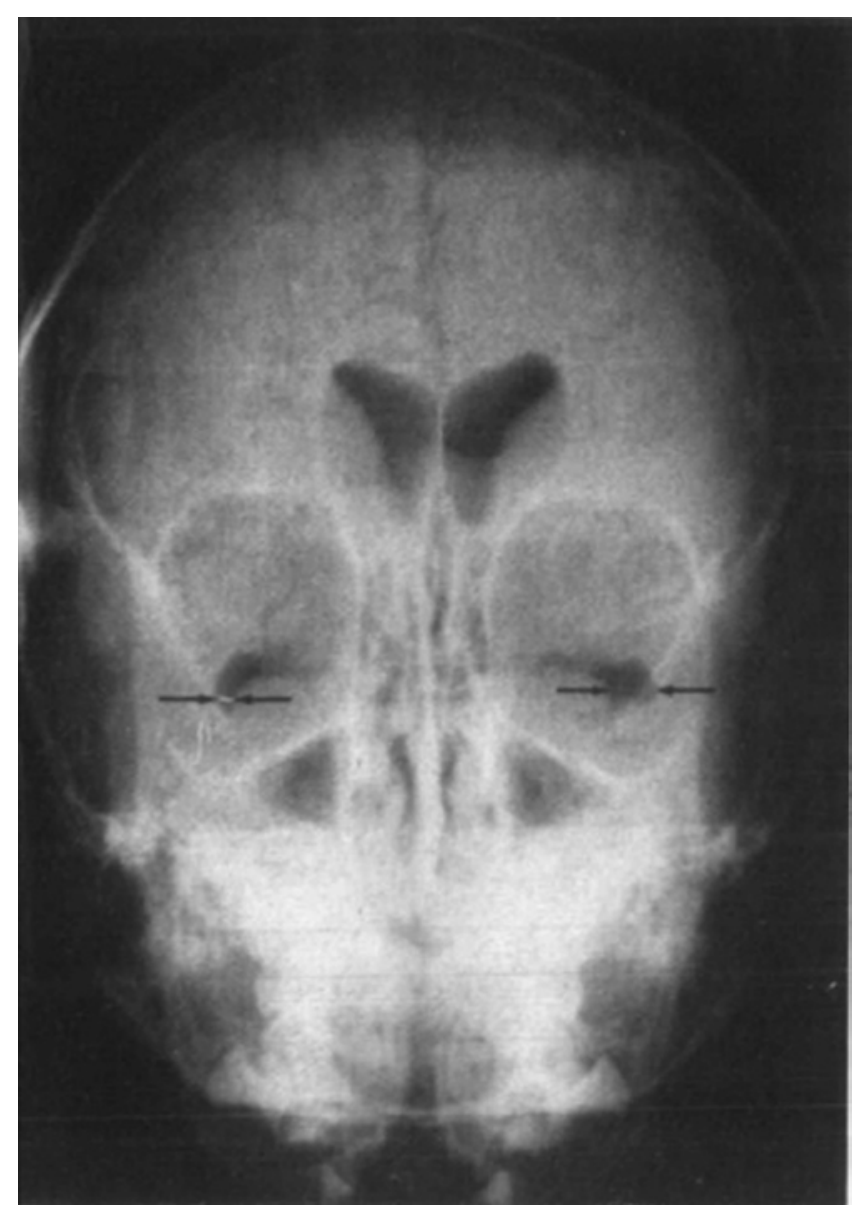

FIG. 5. Pneumoencephalogram, frontal projection, obtained in a child with suspected infantile autism syndrome. The grossly dilated left temporal horn is apparent. Reproduced with permission from Oxford University Press: Hauser SL, DeLong GR, Rosman NP: Pneumographic findings in the infantile autism syndrome. A correlation with temporal lobe disease. Brain 98:667-688, 1975.

years at the University of Breslau. ${ }^{32}$ Furthermore, in a study of 84 patients with posttraumatic epilepsy undergoing PEG, Leksin, a Russian neurosurgeon, reported that $77 \%$ manifested a therapeutic effect to the procedure. This was either through subjective improvement of symptoms or decreased frequency and/or severity of seizures. ${ }^{22}$

Although never broadly recognized for its therapeutic potential, PEG was considered as one of the few options for treating neuropsychiatric diseases in the early-to-mid 20 th century. Existence of an effective intervention for psychiatric conditions helped support the concept that these disorders had a neurobiological basis, thus lending increasing credence to the concept that psychiatric illnesses were based on chemical or structural alterations of the brain. This served in stark contrast to the psychological school of thought, founded in Sigmund Freud's theories of psychopathology, in which mental disease was viewed to be driven by intrinsic or extrinsic aspects of one's personality. ${ }^{21}$ Due to the advent of PEG and its potential for therapeutic benefit, a noticeable increase in other therapeutic attempts and advances for psychiatric conditions was seen in subsequent years. Examples of these methods included insulin- induced comas and convulsions to treat schizophrenia, metrazol-induced convulsions to treat schizophrenia and affective psychoses, and electroconvulsive shock therapy. ${ }^{21}$

\section{Legacy in Popular Culture}

The legacy of PEG today is perhaps best preserved in popular culture, with the most notable depiction of the procedure occurring in the 1973 supernatural horror classic and Academy of Motion Picture Arts and Sciences Award nominee for Best Picture, The Exorcist. The main character, Regan, undergoes the procedure to assess for an intracranial lesion that may explain her demonic behavior. The scene immediately follows the dramatic depiction of cerebral angiography by direct carotid arterial puncture. While a PEG is being performed, Regan is shown restrained on a tilt table with an x-ray tube and detector moving mechanically yet wildly around her. The scene is tense and uncomfortable, largely due to the character's distress from the painful procedure, perhaps contributing to its authenticity. Interestingly, this brief depiction likely provides us with the most readily accessible historical documentation of the procedure. In more modern media programming, PEG was referenced by name in an episode of the popular medical television series House in 2010: it was cited as an example of an archaic and dangerous procedure while working up a case of smallpox. More recently, the 2013 film Jimmy P: Psychotherapy of a Plains Indian featured the procedure.

\section{Conclusions}

Medical, scientific, and cultural aspects of pneumoencephalography provide a rich context for the procedure's place in history and its modern-day legacy. PEG was one of the first methods in medicine to allow reproducible in vivo neuroimaging. It advanced not only our understanding of neuropathology, but also neurophysiology and the management of neuropsychiatric disorders. Despite the novel possibilities offered by PEG, its history cannot be represented as the pure triumph of a successful diagnostic method, largely due to the controversially high morbidity and mortality associated with the procedure. Today, the concept of PEG is certainly not completely antiquated: pneumoencephalography served as the inspiration for myelography and cisternography, which were facilitated by the advent of safe intrathecal contrast agents. Thus, similar to a myriad of other interventions in neuropsychiatry, the history and legacy of pneumoencephalography remain entrenched in a duality between progress and experimentalism.

\section{References}

1. Bakhshi K, Chance SA: The neuropathology of schizophrenia: a selective review of past studies and emerging themes in brain structure and cytoarchitecture. Neuroscience 303:82-102, 2015

2. Bingel A: Encephalographische Erfahrungen. Z Gesamte Neurol Psychiatr 114:323-475, 1928

3. Casamajor L, Laidlaw RW, Kozinn PJ: The technique of pneumoencephalography in children: comparative results with air and oxygen injection. J Pediatr 38:463-467, 1951 
4. Chance SA, Esiri MM, Crow TJ: Ventricular enlargement in schizophrenia: a primary change in the temporal lobe? Schizophr Res 62:123-131, 2003

5. Charash LI, Dunning HS: An appraisal of pneumoencephalography in mental retardation and epilepsy. Pediatrics 18:716-720, 1956

6. Dandy WE: Röntgenography of the brain after the injection of air into the spinal canal. Ann Surg 70:397-403, 1919

7. Dandy WE: Ventriculography following the injection of air into the cerebral ventricles. Ann Surg 68:5-11, 1918

8. Echternacht AP, Campbell JA: Mid-line anomalies of the brain; their diagnosis by pneumoencephalography. Radiology 46:119-131, 1946

9. Flug JA, Lee RS, Giordano M, Cohen SL, Scalcione LR, Irwin GAL, et al: RSNA centennial article: gone but not completely forgotten - pictorial review of "antiquated" radiologic procedures. Radiographics 34:1442-1456, 2014 (Erratum in Radiographics 35:651, 2015)

10. Hauser SL, DeLong GR, Rosman NP: Pneumographic findings in the infantile autism syndrome. A correlation with temporal lobe disease. Brain 98:667-688, 1975

11. Henn FA, Braus DF: Structural neuroimaging in schizophrenia. An integrative view of neuromorphology. Eur Arch Psychiatry Clin Neurosci 249 (Suppl 4):48-56, 1999

12. Hoeffner EG, Mukherji SK, Srinivasan A, Quint DJ: Neuroradiology back to the future: brain imaging. AJNR Am J Neuroradiol 33:5-11, 2012

13. Huber G: Pneumencephalographische und psychopathologische Bilder bei endogenen Psychosen. Berlin: SpringerVerlag, 1957

14. Huber G, Gross G, Schüttler R: A long-term follow-up study of schizophrenia: psychiatric course of illness and prognosis. Acta Psychiatr Scand 52:49-57, 1975

15. Jacobi W, Winkler H: Encephalographische Studien an chronisch Schizophrenen. Archiv Psychiatrie 81:299-332, 1927

16. Jelliffe SE (ed): Book reviews. Frisch, Felix. Die Epilepsie, Biologie, Klinik., Therapie. J Nerv Ment Dis 91:534, 1940

17. Johnstone EC, Crow TJ, Frith CD, Husband J, Kreel L: Cerebral ventricular size and cognitive impairment in chronic schizophrenia. Lancet 2:924-926, 1976

18. Kautzky R, Zülch KJ, Wende S, Tänzer A: Neuroradiology: A Neuropathological Approach. Berlin: Springer, 2012

19. Kawano M, Sawada K, Shimodera S, Ogawa Y, Kariya S, Lang DJ, et al: Hippocampal subfield volumes in first episode and chronic schizophrenia. PLoS One 10:e0117785, 2015

20. Kirkman MA: The role of imaging in the development of neurosurgery. J Clin Neurosci 22:55-61, 2015

21. Kragh JV: Shock therapy in Danish psychiatry. Med Hist 54:341-364, 2010

22. Leksin EN: [Pneumoencephalography in the diagnosis and complex treatment of traumatic epilepsy.] Zh Nevropatol Psikhiatr Im S S Korsakova 81:873-877, 1981 (Russian)

23. Lemke R: Über Ursache und strafrechtliche Beurteilung der Homosexualität: aus der Psychiatrischen und Nervenklinik der Universität Jena. Jena: Fischer, 1940

24. Melchior JC, Dyggve HV, Gylstorff H: Pneumoencephalographic examination of 207 mentally retarded patients. Dan Med Bull 12:38-42, 1965
25. Moriya J, Kakeda S, Abe O, Goto N, Yoshimura R, Hori H, et al: Gray and white matter volumetric and diffusion tensor imaging (DTI) analyses in the early stage of first-episode schizophrenia. Schizophr Res 116:196-203, 2010

26. Müller J, Hermes M, Piepgras U: Adolf Bingel, the second inventor of lumbar pneumencephalography. AJNR Am J Neuroradiol 16:487-490, 1995

27. Nasrallah HA, Jacoby CG, McCalley-Whitters M, Kuperman $\mathrm{S}$ : Cerebral ventricular enlargement in subtypes of chronic schizophrenia. Arch Gen Psychiatry 39:774-777, 1982

28. Olabi B, Ellison-Wright I, McIntosh AM, Wood SJ, Bullmore E, Lawrie SM: Are there progressive brain changes in schizophrenia? A meta-analysis of structural magnetic resonance imaging studies. Biol Psychiatry 70:88-96, 2011

29. Pilowsky LS, Kerwin RW, Murray RM: Schizophrenia: a neurodevelopmental perspective. Neuropsychopharmacology 9:83-91, 1993

30. Rieber I, Sigusch V: Psychosurgery on sex offenders and sexual "deviants" in West Germany. Arch Sex Behav 8:523527,1979

31. Schaldach H: Therapeutische Bedeutung der Encephalographie bei Hirnatrophie [dissertation]. Berlin: University of Berlin, 1942

32. Schleier I: Die therapeutische Wirkung der Encephalographie bei kindlicher Epilepsie. Archiv Psychiatrie 111:200-208, 1940

33. Tondreau RL: Ventriculography and pneumoencephalography: contributions of Dr. Walter E. Dandy. Radiographics 5:553-555, 1985

34. Weinberger DR, Wagner RL, Wyatt RJ: Neuropathological studies of schizophrenia: a selective review. Schizophr Bull 9:193-212, 1983

35. White YS, Bell DS, Mellick R: Sequelae to pneumoencephalography. J Neurol Neurosurg Psychiatry 36:146-151, 1973

\section{Disclosures}

The authors report no conflict of interest concerning the materials or methods used in this study or the findings specified in this paper.

\section{Author Contributions}

Conception and design: all authors. Analysis and interpretation of data: Ishaque, Wallace. Drafting the article: all authors. Critically revising the article: all authors. Reviewed submitted version of manuscript: all authors. Approved the final version of the manuscript on behalf of all authors: Grandhi. Study supervision: Grandhi.

\section{Correspondence}

Ramesh Grandhi, Department of Neurosurgery, University of Texas Health at San Antonio, 7703 Floyd Curl Dr., MC 7843, San Antonio, TX 78229. email: grandhi@uthscsa.edu. 